\title{
L'apprentissage des techniques de communication : attitudes des étudiants en médecine dans un hôpital universitaire au Liban.
}

\author{
Communication Skills Learning: Attitudes of Medical Students \\ of an University Hospital in Lebanon
}

\section{Grace ABI RIZK', Marouan ZOUGHBI ${ }^{1}$}

\begin{abstract}
Résumé Contexte : L'enseignement des techniques de communication (TC) fait partie intégrante du curriculum des facultés de médecine nord-américaines et européennes mais reste rudimentaire dans les facultés libanaises. But: Explorer par une étude transversale les attitudes des étudiants de $\sigma^{\circ}$ et de 7 a année de médecine de l'Université SaintJoseph (USJ) vis-à-vis de l'apprentissage des techniques de communication. Matériels et Méthodes : Un questionnaire anonyme a été administré aux étudiants de la $\sigma^{e}$ et $7^{\circ}$ année de médecine à l'USJ, il comprend 28 questions explorant l'attitude des étudiants envers les TC et permet de calculer un score des attitudes positives et des attitudes négatives. Résultats: Cent quatre étudiants ont participé à l'étude soit un taux de réponse de $91 \%$. Ces étudiants manifestent un intérêt à participer à des cours sur les TC. Ils apprécient l'utilité et considèrent l'absence de tels cours comme une lacune dans leur formation; près de $40 \%$ des étudiants expriment, en revanche, une gêne à participer à des cours de TC et $42 \%$ d'entre eux seulement admettent une difficulté dans la communication. Quarante-six pour cent des étudiants ne peuvent faire confiance à un professionnel non-médecin pour l'apprentissage de ces cours. Le score des attitudes positives retrouvé est de 48,62 \pm 7,6 pour une valeur maximale théorique de 65. Conclusion: Les étudiants trouvent que les $T C$ sont assez importantes et utiles dans leur profession alors qu'elles sont absentes dans leurs programmes. L'apprentissage des TC devrait alors être intégré dans le curriculum de l'Université Saint-Joseph.
\end{abstract}

Mots-clés Education ; techniques de communication ; étudiants ; médical ; attitudes ; apprentissage ; enseignement ; Liban.

Abstract Context: Communication skills (CS) teaching is mandatory in the medical curricula of North American and European universities but remains archaic in Lebanese universities. Purpose: To explore, by means of a transversal study, the attitudes of 6th and 7th year medical students of Saint-Joseph University (SJU) towards communication skills learning. Methods: An anonymous questionnaire was distributed to 6 th and 7 th year medical students of SJU. It included 28 questions exploring the attitudes of students towards CS and allowed to calculate a score of positive and negative attitudes. Results: A hundred and four students completed the questionnaire, which represented a response rate of $91 \%$. These students have manifested an interest to participate to CS courses. They appreciate the usefulness of these courses and consider their absence as a shortcoming in their formation. However, about $40 \%$ of students expressed an inconvenience to attend to CS courses and only $42 \%$ admitted having communication skills difficulties. Forty six percent of students cannot trust a non-medical teacher to learn these courses. The score of positive attitudes was 48,62 \pm 7,6 out of a maximum theoretical value of 65. Conclusion: Students find CS important enough and useful for their profession, while they are not taught in their medical program. CS learning courses must then be integrated in the medical curricula of the SJU.

Keywords Education; communication skills; students; medical; attitudes; learning; teaching; Lebanon. Pédagogie Médicale 2008;9:157-65

1- Département de médecine familiale, Université Saint-Joseph, Centre hospitalier universitaire Hôtel Dieu de France, Beyrouth, Liban Correspondance : Dr Grace Abi Rizk, Département de médecine familiale, Hôtel Dieu de France, Beyrouth, Liban.

Téléphone : +961 1615400 poste : 8191. Télécopie : +961 3 303913. Mailto:gabirizk@yahoo.com 


\section{Recherche et Perspectives}

\section{Introduction}

La communication entre le médecin et son malade est un élément essentiel pour la réussite d'une entrevue médicale ${ }^{1}$. Une communication adéquate augmente la satisfaction $\mathrm{du}$ patient et sa compliance ${ }^{2}$. Lapprentissage des techniques de communication (TC) occupe une place de plus en plus importante dans les programmes d'enseignement de médecine à travers le monde $\mathrm{e}^{3-6}$. Plusieurs organismes professionnels, tel que le "General Medical Council » (GMC) britannique ou plusieurs sociétés savantes, telle que la Fédération mondiale de l'éducation médicale, recommandent l'intégration de cours sur les TC dans le curriculum médical ${ }^{7,8}$.

L'attitude des étudiants en médecine face à l'apprentissage des TC a été explorée dans plusieurs études de la littérature médicale, ${ }^{90}$. Au Liban, cet apprentissage ne faisait pas partie, jusqu'à la date du présent travail, des programmes des facultés de médecine. Cependant à l'"American University in Beirut " (AUB), l'enseignement des TC consiste à exposer les étudiants en cinquième année de médecine à une série de consultations médicales filmées, suivies de discussions sur les bonnes et les mauvaises techniques de communication. Un entraînement pratique aux TC ne se fait d'une façon formelle que dans le département de médecine familiale de l'université Saint-Joseph à Beyrouth.

Cette étude, effectuée conjointement à une autre portant sur l'attitude des médecins de la faculté de médecine Saint-Joseph quant à l'enseignement des TC (manuscrit en préparation), vise à concevoir l'intégration de l'enseignement des TC dans cette université. Une proposition de cours en découlera et sera intégrée dans la formation médicale à la faculté Saint-Joseph après approbation des instances concernées.

L'objectif primaire de notre étude est d'explorer les attitudes des étudiants libanais, à la fin de leurs études médicales, face à l'apprentissage des TC. La recherche de facteurs prédictifs de ces attitudes constitue un objectif secondaire.

Nos hypothèses sont les suivantes : a) les étudiants surestiment leur capacité de communication avec les patients ; b) les étudiants ont, cependant, des attitudes positives quant à l'apprentissage des TC.

\section{Matériel et méthodes}

\section{Recrutement}

Il s'agit d'une étude transversale comportant un question- naire rempli par les étudiants. Ce questionnaire a été adminsitré lors des conférences hebdomadaires, durant le mois de juin 2004, aux étudiants de sixième et septième année de médecine en stage à l'hôpital Hôtel-Dieu de France (hôpital universitaire affilié à l'Université SaintJoseph), Beyrouth, Liban. Les étudiants en sixième année sont au début de leurs stages cliniques, ceux de septième année en ont déjà bénéficié pendant un an. Tous les étudiants sont francophones.

\section{Questionnaire}

Le questionnaire est adapté de celui employé dans une étude publiée par Rees et al. en 2002 (communication skills attitude scale: $C S A S)^{11}$ et traduit en français puis retraduit en anglais pour vérifier la validité de la traduction.

Le questionnaire est anonyme, ses données individuelles se limitant à la précision du genre et de l'année d'étude de chaque participant. Après une brève introduction sur les TC, il inclut 28 propositions dont 26 tirées de l'étude de Rees et al. et deux supplémentaires (27 et 28), ajoutées par les auteurs. Ces deux propositions visent à rechercher l'exposition des étudiants à un enseignement des TC durant la période des cours et des stages cliniques.

L'évaluation est faite sur une échelle de Likert à cinq niveaux de réponse, le premier correspondant à un désaccord complet et le cinquième à un accord complet. L'analyse faite dans l'étude de Rees et al. ${ }^{11}$ permet de regrouper les réponses en deux scores. Le score des attitudes positives (SAP) envers l'apprentissage des TC (comme : « développer mes techniques de communication est aussi important que développer mes connaissances médicales ") est calculé en additionnant les scores des items 4, 5, 7, 9, 10, 12, 14, 16, 18, 21, 22, 23 et 25 avec une bonne consistance interne $(\alpha=0,873)$. Le score des attitudes négatives (SAN) (comme: «je ne peux être dérangé pour assister à des séances d'apprentissage aux TC») est calculé à partir des items $1,2,3,6,8,11,13,15$, $17,19,20,24$ et 26). La consistance interne de ce score est également satisfaisante $(\alpha=0,805)$. A noter que les scores des items 1 et 22 ont été inversés avant d'être inclus dans le calcul des scores comme il a été suggéré par l'analyse de facteurs du CSAS. Chaque score, composé de 13 questions, a ainsi des valeurs comprises entre 13 et 65 .

\section{Analyse statistique}

L'analyse statistique a été faite en utilisant le logiciel stata version 7.0. La normalité des distributions a été testée. Les variables catégoriques ont été présentées en pourcentages et les variables continues en moyenne et déviation standard. Le test de la loi normale a été utilisé 
pour les variables continues à distribution normale et le test de chi $^{2}$ pour la comparaison des variables qualitatives.

\section{Résultats}

Cent quatorze étudiants (57 en sixième et 57 en septième année) ont été sollicités pour participer à l'étude ; 104 ont accepté, soit un taux de réponse de l'ordre de $91 \%$. Dix étudiants n'ont pas répondu, parmi lesquels huit n'étaient pas au Liban durant la période de l'étude et deux ont refusé de remplir le questionnaire sans en préciser les raisons. Cinquante-quatre étudiants sont en sixième année de médecine (52\%) et 50 (48 \%) étudiants sont en septième année. Le sexe féminin représente $34 \%$ de l'ensemble des étudiants. La distribution des sexes est comparable dans les deux années d'études.

\section{Le score des attitudes positives}

La valeur moyenne de ce score sur l'ensemble de la population de l'étude est de 48,62 \pm 7,6 (29-63). L'analyse de la distribution de ce score montre qu'il suit une distribution normale. L'analyse du score en fonction du sexe des étudiants et de leur année d'étude ne montre pas une différence significative ( $\mathrm{p}=0,7$ et $\mathrm{p}=0,82$ respectivement) (Tableau 1).

\section{Le score des attitudes négatives}

La valeur moyenne de ce score est de 33,06 \pm 4,8 (2150). La distribution suit également une distribution normale. L'analyse montre un score des attitudes négatives plus marqué chez les femmes $(33,74$ vs 31,74$)$ avec une différence significative $(\mathrm{p}=0,04)$ alors qu'il est comparable dans les deux années d'études $(\mathrm{p}=0,47)$ (Tableau 1).

Les réponses aux questions 27 et 28 sont analysées séparément. Elles montrent une majorité en accord avec ces deux propositions et donc à l'absence d'apprentissage. Les résultats des réponses aux questions 27 et 28 ont par la suite été regroupés en deux catégories : l'une (catégorie 1) pour les réponses de niveaux 1 et 2 (complètement en désaccord et en désaccord) et l'autre (catégorie 2) pour les réponses de niveaux 3, 4 et 5 (neutre, en accord et complètement en accord). Les étudiants de la catégorie 1 ont reçu un apprentissage de TC durant les cours ou les stages alors que les étudiants de la catégorie 2 affirment ne pas en avoir reçu. La comparaison entre les années d'étude montre que les étudiants de septième année n'ont pas été davantage confrontés à l'apprentissage des TC durant leurs cours théoriques que ceux de sixième année $(14 \%$ vs $5,56 \% ; \mathrm{p}=0,08)$.

\begin{tabular}{|l|c|c|c|c|c|c|}
\hline \multicolumn{5}{|c|}{$\begin{array}{c}\text { Tableau 1 : } \\
\text { Scores des attitudes positives (SAP) et négatives (SAN) à l'égard de la formation } \\
\text { à la communication médecin-patient, recueillis chez les étudiants de l'étude }\end{array}$} \\
\hline & \multicolumn{3}{|c|}{ SAP } & \multicolumn{3}{c|}{ SAN } \\
\hline & moyenne & écart type & p & moyenne & écart type & p \\
\hline Année d'étude & 48,625 & 7,6 & & 33,06 & 4,8 & \\
\hline sixième & 48,46 & 7,8 & & 32,74 & 4,68 & \\
\hline septième & 48,8 & 7,47 & 0,7 & 33,42 & 4,92 & 0,47 \\
\hline Sexe & & & & & & \\
\hline homme & 49,03 & 6,81 & & 31,74 & 4,51 & \\
\hline femme & 48,42 & 8,02 & 0,82 & 33,74 & 4,83 & 0,04 \\
\hline
\end{tabular}




\section{Recherche et Perspectives}

En revanche, les étudiants de la septième année ont reçu plus de formation pratique durant les stages que ceux de la sixième année (16\% vs 5,56\%; $\mathrm{p}<0,11)$.

La comparaison des SAP et SAN en fonction des réponses à la question 27 (" personne ne m'a appris des TC durant mes cours théoriques ") ne montre pas de différence significative entre les différents sous groupes $(p=0,65$ et $\mathrm{p}=0,12$ respectivement). Une même comparaison en fonction des réponses à la question 28 («personne ne m'a appris des TC durant mes stages cliniques ») n'a pas montré de différence significative $(\mathrm{p}=0,89$ et $\mathrm{p}=0,41$ respectivement). Un regroupement des 26 réponses en trois catégories (catégorie 1 pour les réponses de niveaux 1 ou 2 , catégorie 2 pour les réponses de niveau 3 , catégorie 3 pour les réponses de niveaux 4 ou 5) a été fait. Le tableau 2 représente la distribution des réponses des participants.

\section{Discussion}

Notre étude montre la présence d'attitudes positives chez les étudiants de l'Université Saint-Joseph vis-à-vis de l'apprentissage des TC. Ces étudiants manifestent un intérêt à participer à des cours sur les TC. Ils en apprécient l'utilité et considèrent l'absence de tels cours comme une lacune dans leur formation.

Quarante pour cent des étudiants expriment, en revanche, une gêne à participer à des cours de TC. Quarante-six pour cent des étudiants ne peuvent faire confiance à un professionnel non-médecin pour l'apprentissage de ces aspects et $77 \%$ des étudiants participant à l'étude préferent apprendre les TC dans le cadre d'un sujet médical. Le SAP trouvé est de 48,62 \pm 7,6 pour une valeur maximale théorique de 65 . Le SAN est de 33,06 $\pm 4,8$ et est significativement plus élevé chez le sexe féminin.

Les expériences non formelles d'apprentissage des TC auxquelles ont été exposés $11 \%$ des étudiants n'ont pas influencé leurs attitudes positives ou négatives envers les TC. Finalement, notons que $42 \%$ des étudiants seulement admettent une difficulté dans la communication.

Les avantages de l'introduction des cours de TC ont fait l'objet de plusieurs études rapportées dans la littérature médicale. Ces cours améliorent la qualité de la relation entre le médecin et le patient et la performance des médecins amenés à travailler en équipe ${ }^{12}$; par ailleurs ces cours offrent la possibilité de donner un feedback aux étudiants, leur permettant de mieux évaluer leur capacité à communiquer et de la développer ${ }^{13}$. L'amélioration de la qualité de la communication du médecin avec le patient augmente à son tour la satisfaction du dernier et sa compliance au traitement institué.

L'apprentissage des TC revêt, de par le monde, des formes très variables et donne des résultats également variables ${ }^{14}$. La littérature internationale fait apparaître que les méthodes expérientielles sont supérieures aux approches purement théoriques ${ }^{15}$. Une étude menée en Grande-Bretagne montre la présence d'une grande différence dans le contenu, la durée, l'année d'administration et la méthode d'évaluation des apprentissages selon les différentes universités, ce qui rend les réactions des étudiants à ce type de cours assez hétérogènes.

Nos résultats rejoignent ceux de la littérature. Les recommandations faites par la "Word Federation for Medical Education - WFME - task force " sont en faveur de l'apprentissage des TC comme partie intégrante du programme de médecine. En plus, Rosenthal et Ogden ont mené une étude visant à explorer les attitudes des étudiants de la "Royal free hospital school of medicine " ${ }^{10}$. Dans cette étude 64,8 \% des étudiants ont répondu en accord avec l'affirmation : " une plus grande importance doit être accordée aux techniques de communication ». La comparaison de nos résultats avec ceux rapportés dans l'étude de Rees et al. ${ }^{16}$, fait apparaitre un SAP comparable à celui colligé auprès des étudiants des universités de Nottingham $(48,5)$ et de Leicester $(52,38)$. Dans l'étude de Rees et al., la variable " âge " est inversement corrélée au SAP. Dans notre étude, l'âge moyen des étudiants est supérieur à celui des étudiants de ce travail mais l'attitude positive est similaire. La persistance de la demande en dépit de l'âge peut être expliquée par l'absence d'enseignement des TC dans les universités libanaises et par la demande croissante en la matière lors de la confrontation à des patients mais ceci reste à documenter par des études ultérieures. Le score des attitudes négatives trouvé dans notre étude est légèrement supérieur à celui rapporté dans l'étude de Rees et al. ${ }^{16}$. Le SAN est significativement plus élevé chez les femmes, constatation qui ne correspond pas aux données retrouvées dans la littérature ${ }^{17}$.

La présence d'attitudes négatives chez les étudiants pourrait évoquer une priorité accordée à l'apprentissage des dimensions biomédicales des problèmes de santé et une surévaluation de leurs acquis antérieurs en matière de communication ou encore un manque de motivation à approfondir le sujet ${ }^{18}$. 


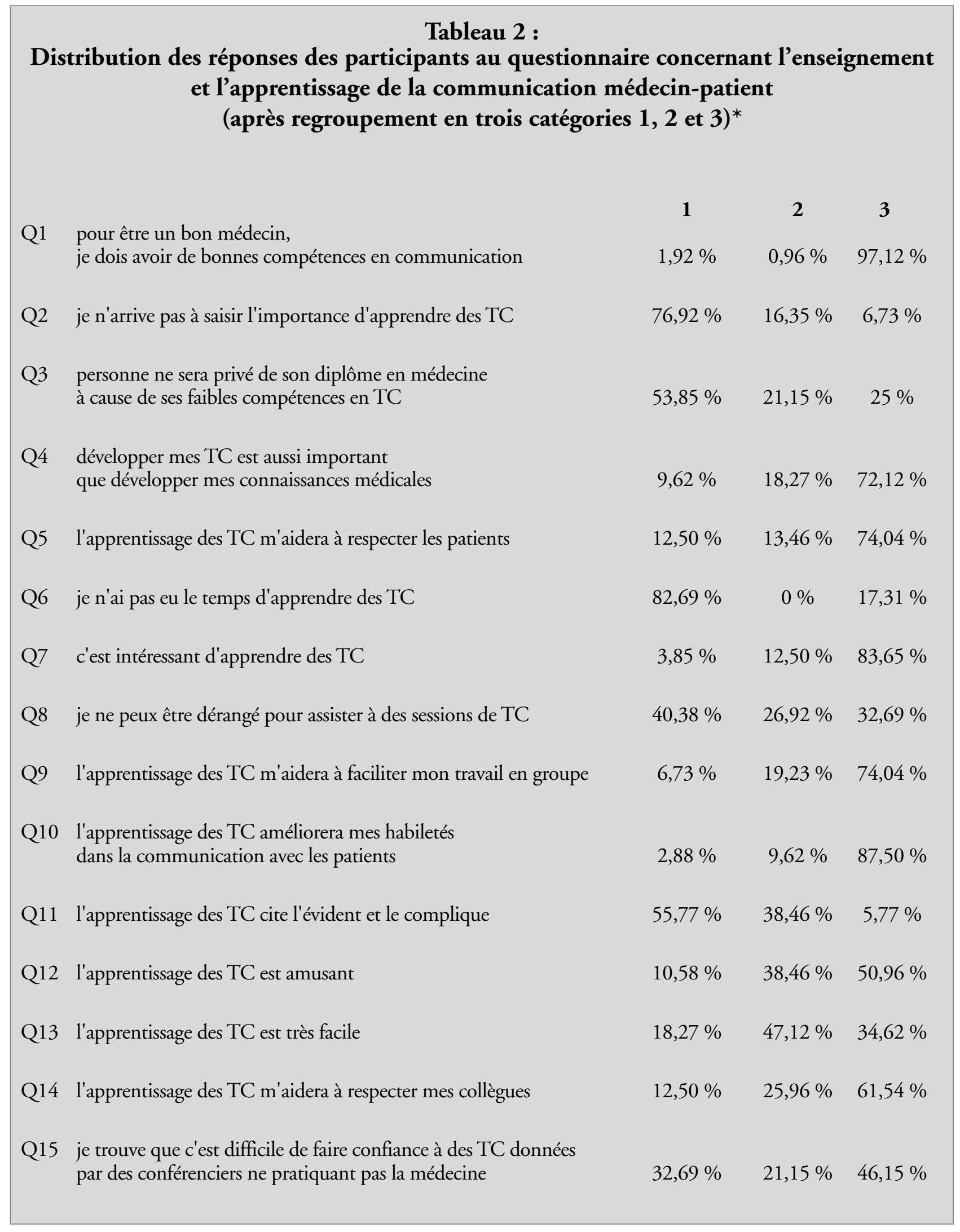




\section{Recherche et Perspectives}

\section{Tableau 2 (suite)}

Q16 l'apprentissage des TC m'aidera à reconnaittre les droits

Q17 l'enseignement des TC aurait un meilleur impact s'il est pratiqué dans le cadre d'un sujet médical

Q18 quand j'avais appliqué pour les études médicales, je pensais que l'apprentissage des TC était vraiment une bonne idée

Q19 je n'ai pas besoin de bonnes techniques de communication pour être un médecin

Q20 je trouve qu'il m'est difficile d'admettre que j'ai des problèmes avec mes $\mathrm{TC}$

Q21 je pense que c'est vraiment utile d'apprendre les TC durant mes études médicales

Q22 ce sont plutôt mes habiletés à réussir les examens que mes habiletés de communication qui me font passer les examens de médecine

Q23 l'apprentissage des TC fait partie de l'apprentissage de la médecine

Q25 l'apprentissage des TC est important car mes habiletés de communication sont des habiletés que je garde pour toute la vie

Q26 l'apprentissage des TC doit être réservé aux étudiants de psychologie plutôt qu'aux étudiants de médecine

* $1=$ complètement en désaccord + en désaccord ; $2=$ opinion neutre ; 3=complètement en accord + en accord. $T C$ : techniques de communication 
Cependant en l'absence de cours formels et obligatoires de TC dans le cursus médical de notre faculté et en l'absence d'évaluation des capacités de communication des étudiants, cet apprentissage est mis en second plan et amène les étudiants à ne pas y accorder du temps. D'autre part, l'intégration de l'enseignement des TC au curriculum médical peut poser pour certains des difficultés concernant la personne enseignante ou la méthode avec laquelle ce cours est abordé, surtout lors de l'entraînement pratique ${ }^{19}$. Le modèle de rôle constitue un moteur très puissant pour l'apprentissage d'habilités telle que la communication. Des stratégies devraient être mises en place pour faciliter l'émergence parmi les cliniciens des personnes ressources pour l'enseignement de la communication professionnelle ${ }^{20}$. L'absence d'un effet de l'apprentissage des TC, durant les cours et les stages, sur les scores des attitudes peut être expliqué par le fait que son importance dans la prise en charge des patients n'a jamais été explicitée aux étudiants. En plus, des rétroactions n'ayant pas eu lieu, il est difficile aux étudiants de noter le progrès et l'utilité de cet apprentissage. Les étudiants ne réalisent pas que les habitudes acquises durant les années de formation, qu'elles soient bonnes ou mauvaises, ne peuvent être modifiées que par des stratégies d'enseignement et d'apprentissage spécifiques, incluant des rétroactions répétées, et qu'il est essentiel en médecine tout au long de la vie professionnelle de consolider leurs habiletés à travers des applications répétées et des rétroactions immédiates $^{20}$. Le fait que les étudiants n'admettent pas un déficit en matière de techniques de communication peut constituer un obstacle à la mise en place d'un enseignement dédié à ces dimensions. Les études montrent d'ailleurs que les auto-évaluations par les étudiants sont faiblement corrélées avec les évaluations externes ${ }^{21-23}$ et que les connaissances théoriques en TC ne permettent pas une communication réussie avec les patients. Ces données de la littérature remettent en cause la réalité des capacités des étudiants libanais en matière de communication et plaident en faveur d'études ultérieures comportant une évaluation par des tiers, ainsi que des évaluations de la satisfaction des patients.

La présente étude constitue une première en matière d'évaluation des attitudes envers les TC au liban. Le questionnaire utilisé est validé sur une population d'étudiants en médecine comparable en certains points aux étudiants de notre étude, ce qui, selon nous, le rend applicable à notre population. Le taux de participation élevé est un élément à mettre au crédit de la validité des résultats. Certaines limites rendent cependant la généra- lisation des résultats aux autres universités libanaises difficile. Au total, d'autres études seraient utiles pour clarifier certaines questions que cette étude a soulevées. Une évaluation objective des capacités de communication des étudiants est nécessaire. Des études prospectives évaluant ces capacités avant et après l'instauration des cours sont utiles et recommandées ${ }^{24}$ et un suivi des attitudes des étudiants par le CSAS serait utile pour évaluer et ajuster l'apprentissage des TC.

\section{Conclusion}

Cette première étude faite au Liban concernant les attitudes des étudiants de médecine vis-à-vis des TC met en évidence des attitudes positives envers l'apprentissage de ces cours, bien que certaines attitudes négatives soient notées. Un déficit en la matière est clairement constaté. Nous pensons dès lors que l'intégration de l'enseignement des TC dans le programme de médecine s'avère nécessaire.

\section{Contributions}

Grace Abi Rizk a conçu le protocole de recherche et rédigé les versions initiales et révisées du manuscrit. Marouan Zoughbi a participé à l'interprétation des résultats et à l'écriture du manuscrit. 


\section{Recherche et Perspectives}

\section{Références}

1. Tai Pong LAM. What kind of communication skills do Chinese fishermen expect from their family doctors? Asia Pacific Family Medicine 2002;1:83-7.

2. Wagner PJ, Lentz L, Heslop SD. Teaching communication skills: a skills-based approach. Acad Med 2002;77:1164.

3. Anderson L \& Sharpe P. Improving patient and provider communication: a synthesis and review of communication interventions. Patient Education and Counselling 1991;17:99-134.

4. Badenoch J. Communication skills in medicine: the role of communication in medical practice. JR Soc Med 1986;79:565-7.

5. Bensing J \& Dronkers J. Instrumental and affective aspects of physician behavior. Med Care 1992;30:283 98.

6. Davis $H$ \& Fallowfield L. Counselling and Communication in Health Care. London: Wiley, 1991.

7. General Medical Council. Tomorrow's doctors:Recommandations on Undergraduate Medical Education. London: GMC, 2003. [Online]. Disponible sur : http://www.gmc-uk.org/education/undergraduateltomdoc.pdf

8. The Executive Council. Word Federation for Medical Education Task Force on Defining International Standards in Basic Medical education. Report of the Working Party, Copenhagen. Med Educ 2000;34:665-75.

9. Walker $L G$, Haldane JD, Alexander DA. A medical curriculum: evaluation by final-year students. Med Educ 1981;15:377-82.
10. Rosenthal J, Ogden J. Changes in medical education: the beliefs of medical students. Med Educ 1998; 32:127-32.

11. Rees $C$, Sheard C, Davies S. The development of a scale to measure medical students' attitudes towards communication skills learning: the Communication Skills Attitude Scale (CSAS). Med Educ 2002;36:141-47.

12. Ang M. Advanced communication skills: conflict management and persuasion. Acad Med $2002 ; 77: 1166$.

13. Millis SR, Jain SS, Eyles M, Tulsky D, Nadler SF, Foye PM et al. Assessing physicians' interpersonal skills: do patients and physicians see eye-to-eye? Am J Phys Med Rehabil 2002;81:946-51.

14. Hargie O, Dickson D, Boohan M, Hughes K. A survey of communication skills training in UK Schools of Medicine: present practices and prospective proposals. Med Educ 1998;32 :25-34.

15. Humphris GM, Kaney S. The Objective Structured video examination for assessment of communication skills. Med Educ 2000;34:939-45.

16. Rees $C$, Sheard C. The relationship between medical students' attitudes towards communication skills learning and their demographic and education-related characteristics. Med Educ 2002;36:1017-27.

17. Batenburg V, Smal JA. Does a communication skills course influence medical students' attitudes? Med Teach 1997;19:263-9.

18. Boulé R, Gerard G, Clavet D, Bernier C. Stratégies d'animation de groupe sur la communication patient-médecin-famille. Pédagogie Médicale 2000;1:13-22 
19. Yoshida T, Milgrom P, Coldwell S. How do U.S. and Canadian dental schools teach interpersonal communication skills? J Dent Educ 2002;66:1281-8.

20. Millette B, Lussier MT, Goudreau J. L'apprentissage de la communication par les médecins : aspects conceptuels et méthodologiques d'une mission académique prioritaire. Pédagogie médicale 2004;5:11026.

21. Aspegren K. Teaching and learning communication skills in medicine: a review with quality grading of articles, Med Teach 1999;21:563-70.
22. Marteau TM, Humphrey C, Matoon G, Kidd J, Lloyd $M$, Horder J. Factors influencing the communication skills of first-year clinical medical students. Med Educ 1991;25:127-34.

23. Farnhill D, Hayes SC, Todisco J. Interviewing skills: self evaluation by medical students. Med Educ 1997;31:122-7.

24. Wilson BE. Performance-based assessment of internal medicine interns: evaluation of baseline clinical and communication skills. Acad Med 2002;77:1158.

Manuscrit reçu le 16 octobre 2007 ; commentaires éditoriaux formulés aux auteurs le 27 juin 2008 ; accepté pour publication le 7 juillet 2008. 